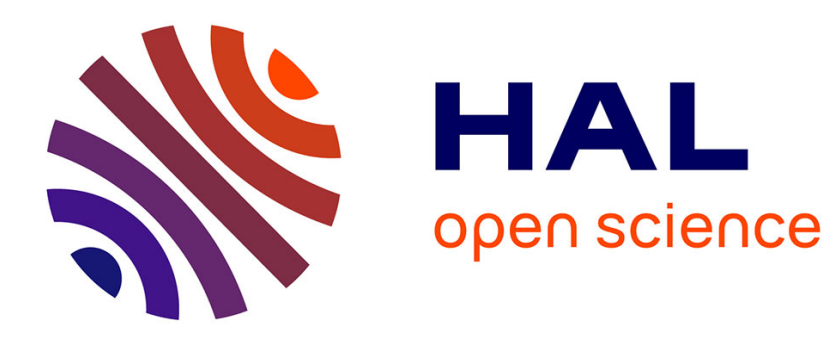

\title{
Le décoratif, emblème du malaise fin-de-siècle dans Chérie
}

\author{
Dominique Pety
}

\section{To cite this version:}

Dominique Pety. Le décoratif, emblème du malaise fin-de-siècle dans Chérie. Cahiers Edmond et Jules de Goncourt, 2009, Les romans d'Edmond, 1 (16), pp.73-84. 10.3406/cejdg.2009.1018 hal-02467862

\section{HAL Id: hal-02467862 \\ https://hal.univ-smb.fr/hal-02467862}

Submitted on 5 Feb 2020

HAL is a multi-disciplinary open access archive for the deposit and dissemination of scientific research documents, whether they are published or not. The documents may come from teaching and research institutions in France or abroad, or from public or private research centers.
L'archive ouverte pluridisciplinaire $\mathbf{H A L}$, est destinée au dépôt et à la diffusion de documents scientifiques de niveau recherche, publiés ou non, émanant des établissements d'enseignement et de recherche français ou étrangers, des laboratoires publics ou privés. 


\section{Le décoratif, emblème du malaise fin-de-siècle dans Chérie}

Faire le roman d'un éventail, tel est le projet qu'Edmond de Goncourt esquisse en 1864 dans le Journal', et c'est celui qu'il semble avoir réalisé vingt ans plus tard avec Chérie (1884). Le romancier illustre ainsi l'évolution du genre, avec le renouvellement thématique qu'il préconisait dans la préface des Frères Zemganno (le réalisme doit changer d'objet et décrire aussi, après le bas peuple, le grand monde), il souligne aussi l'atrophie qui a gagné le récit de fiction, où l'action s'est réduite, où l'attention s'est déportée de l'humain vers l'objet'.

Mais Chérie, dernier roman du dernier Goncourt, ne correspond pas seulement à une nouvelle façon de faire (ou de ne plus faire) du roman, il illustre aussi une nouvelle manière de faire (ou de ne plus faire) de l'histoire. On sait, depuis l'époque de Germinie Lacerteux, que le roman est l'histoire du présent, qu'il s'appuie sur des documents (la préface des Frères Zemganno démultiplie à l'excès ce motif, et Edmond, par un appel aux lecteurs, a encore élargi son mode de prospection pour le présent roman). Mais sur Chérie ne pèse pas l'érudition voyante, poussiéreuse, affichée jusque dans le titre de ses monographies sur le XVIII ${ }^{c}$ siècle. Comme Octave Uzanne qui a justement publié deux ans plus tôt (1882) une histoire de l'éventail et qui insiste sur l'essentielle légèreté du propos (à l'image de son objet), Edmond affiche ici une esthétique du vaporeux, en adéquation avec son personnage de jeune fille, noyée dans les parfums et les chiffons, de sorte que la frivolité apparente du sujet semble d'emblée déconsidérer la visée historique.

Le décoratif ainsi exhibé au premier plan est, on le voit, l'emblème d'un malaise. Il est peut-être d'abord le reflet d'une mutation profonde qui s'amorce dans la sphère culturelle et sociale, où l'essor de la vie privée, l'importance croissante conquise par l'intérieur bourgeois, vont consacrer le rôle des femmes. À elles s'adressent nombre des ouvrages d'Uzanne, ce sont elles que sollicite Edmond de Goncourt pour l'écriture de son dernier roman, et les objets de leur toilette occupent désormais le premier plan ${ }^{3}$. Cette féminité omniprésente est sentie comme un mystère qu'il s'agit de percer, et qu'on s'efforcera de cerner sous une dénomination appropriée, le fameux néologisme de féminilité. Elle

1. Vuir Chérie, éd. J.-L. Cabanès et Ph. Hamon, Éd. La chasse au Snark, 2002, préface p. 30.

2. Voir sur ce point mon essai Collection et décoration au XIX siècle. Du document de l'historien au bibelot de l'esthète, à paraître aux Presses universitaires de Paris-Ouest.

3. Dans la démarche du collectionneur aussi : Edmond de Goncourt acquiert désormais des "petits objets à l'usage de la femme " à partir de la fin des années 1880. 
renvoie au corps, à ses incessantes métamorphoses, qui se liront dans l'évolution du costume, dans le rapport à la toilette, de l'enfance à la sexualité, de la croissance à la maladie; mais ce corps qui mûrit est celui d'un être qui s'observe autant qu'on l'observe, et les mutations physiologiques font signe vers un clivage psychologique, que le rapport à la parure aura aussi pour tâche d'exacerber. Cette instabilité profonde n'est finalement pas tant celle de l'autre sexe, dont on s'efforce de contenir la puissance latente (on le verra au ton souvent péremptoirc de l'écrivain), que celle de l'individu fin-de-siècle, fragilisé et qui cherche refuge dans ses objets, lesquels sont bien dépositaires de son malaise.

\section{Décors}

A la différence des inventaires exhaustifs de La Maison d'un artiste (1881), mais aussi des intérieurs soignés de cette autre artiste qu'est La Faustin (1882)', les descriptions de lieux esthétiquement élaborés sont peu nombreuses dans Chérie, où la priorité est donnée aux costumes. En outre, on remarque que ces descriptions elles-mêmes concourent précisément à reporter l'attention de l'enveloppe architecturale à cette autre enveloppe, qui serre le corps de plus près et qui relève aussi d'un haut degré d'élaboration, la toilette.

La chambre parisienne de Chérie jeune fille, évoquée succinctement, donne surtout à voir un tissu d'ameublement très travaillé :

Le mobilier de la chambre, un ancien mobilier du Premier Empire, est tout à fait jeune fille. Ce sont des fauteuils, des chaises en acajou, avec des coussins volants en piqué blanc, où l'on voit, brodés très en relief et également en blanc, deux cols de cygne accolés au dessus d'un grand chiffre, qui par une coöncidence bizarre se trouve être un $\mathrm{C}^{2}$.

En outre, ce mobilier raffiné est surtout un décor dans lequel on veille à sa toilette et à celle des amies en visite (" des causeries chuchotantes et doucement rieuses, au milieu desquelles celle qui écoute, d'un adroit et preste geste tenant de la caresse, donne de temps en temps pour la faire bouffer, un petit coup sur la jupe de celle qui parle"), et où l'on discute des modes du moment ("Les élégantes fillettes sont en train de discuter un chapeau de Laure, porté la semaine dernière par $\mathrm{M}^{\mathrm{mc}}$ Tony-Freneuse ") ; cependant, par un brutal contraste, tout le monde rit néanmoins à une anecdote scatologique, comme si le corps naturel signalait énergiquement sa présence sous le corps paré.

1. Voir aussi, l'année où paraît Chérie, les décors d'A rebours (Edmond de Goncourt voir d'ailleurs en Des Esseintes le " futur " de Chérie!)

2. Chérie, op. cit., chap. LXII, p. 206. 
L'élaboration du décor, censé conjuguer élégance et recherche du confort', débouche aussi sur l'évocation d'un malaise du corps :

Chérie, après le déjeuner, était remontée chez elle, et allongée sur sa chaise longue, dans la pénombre tiède de la chambre aux volets fermés, un doigt au milieu d'un livre qu'elle n'avait pas le courage de continuer à lire, indolemment somnolente, les yeux mi-clos, elle aspirait, avec de petits frémissements voluptueux des narines, l'odeur des orangers de la cour d'honneur, jetant dans le moment des parfums entêtants ${ }^{2}$.

Chérie n'est pas ici dans son hôtel parisien du Ministère de la Guerre, mais dans la propriété provinciale du Muguet, fastueusement réaménagée par son grand-père; après le chapitre qui évoque l'architecture extérieure, la "cour d'honneur digne d'un petit Marly", Chérie apparaît plus sobrement dans la pose de la dame au sofa, selon un motif que l'on retrouve à diverses reprises dans le roman, et qui suscite irrésistiblement l'image de Juliette Récamier, tant abondent par ailleurs les références au Premier Empire ${ }^{3}$. Ce modèle historique, dont Chérie se rapproche aussi par son goût quasi exclusif du blanc, légitimera l'héroïne dans son rôle de reine de la mode et du goût.

On retrouve, dans la description d'un autre intérieur, la même pose maladive, explicitement associée à l'élégance et au confort d'un lieu explicitement conçu cette fois comme une robe de grand couturier :

$M^{\text {mec }}$ Tony-Freneuse recevait ses amis dans un petit salon octogone, aux murs et au plafond tendus de satin, et où ne se voyait pas un seul bibelot, mais où le mobilier, fabriqué par le premier tapissier de la capitale, était un pur chefd'œuvre dans l'ordre de ces mobiliers du XIX siècle, qui ne sont que capitonnage et rond et mol et élégant contournement d'étoffe, que n'arrête et ne termine jamais la bordure sèche d'un bois.

La tenture, dont elle l'avait fait habiller, ce petit salon, selon l'expression de la maîtresse de maison, présentait une série de panneaux brodés sous la direction de Worth, et qui, disait-on, lui avaient coûté 60.000 francs. [...]

La jolie $\mathrm{M}^{\mathrm{mi}}$ Tony-Freneuse était couchée sur une chaise longue, que la jardinière à compartiments entourait de trois côtés, comme d'une espèce d'alcôve

1. Après le mobilier anguleux des styles Empire puis néo-gothique, on sait que le capitonnage s'impose au milieu du Second Empire (Chérie meurt en 1870 à 19 ans). Voir plus loin le salon de $M^{\text {me }}$ Tony-Freneuse.

2. Ibid., chap. XIVIII, p. 164.

3. Les Goncourt ont évoqué dans leur Histoire de la Société française sous le Directoire (1855) la chambre de $\mathrm{M}^{\mathrm{mc}}$ Récamier sous le Consulat, dans son hôtel fastueusement décoré de la rue du Mont-Blanc (Flammarion-Fasquelle [1929], chap. I, p. 55-56). Voir ma communication " $\mathbf{M}^{\mathrm{mc}}$ Récamier et la décoration d'intérieur " au colloque Juliette Récamier dans les arts et la littérature. La fabrique des representations, sous la direction de Sarga Moussa et Delphine Gleizes, Musée des Beaux-Arts de Lyon, 15-16 mai 2009 (à paraître). 
de verdure, fleurie d'une dizaine de camélias, à la nuance rose de Chine, se détachant sur le satin bleu de ciel de la tenture et des meubles'.

C'est aussi le dispositif décoratif plus précis de la jardinière de camélias qui évoque l'étoffe fleurie d'une robe : un motif similaire est d'ailleurs apparu au chapitre précédent pour la toilette de Chérie (" une robe bleu de lapis, un lampas semé de deux boutons de rose à la queue entrecroisée $\left.[\ldots]^{2} »\right)$.

\section{Le corps et la toilette}

Les décors conduisent donc bien à la parure des corps, mais ceux-ci entretiennent avec la toilette une relation complexe, soit que le corps maladif semble à l'unisson d'un vêtement raffiné, soit que sa vive exubérance forme à l'inverse un contraste brutal.

Très proches de Baudelaire pour qui la mode doit être considérée " comme un symptôme du goût de l'idéal " et une négation de la nature grossière, Edmond de Goncourt fait ici de la toilette une activité artistique parfaitement maîtrisée et qui tend à la sublimation du corps ${ }^{3}$. Le chapitre LXXVIII, qui décrit les après-midis solitaires de Chérie dans des toilettes de son invention, est presque une apothéose en chambre : si, deux chapitres auparavant, Edmond a déjà évoqué "la spiritualité du chiffon", avec "l'emploi presque unique d'étoffes de nuage et de vapeur " $"$, cette fois, dans une longue phrase au rythme ascendant, il suggère presque la divinité de ce corps métamorphosé par le costume :

À ce moment de son existence toute donnée au façonnement de son être en une figuration d'élégance, et de l'élégance portée au dernier raffinement, dans l'élaboration coquette de cette gloire, pour ainsi dire, apportée par la toilette au corps et au visage de la femme, parmi des aspirations féminines à chercher, dans les fantaisies de la mise et de la parure, une jolie et radieuse surhumanité $[\ldots]^{5}$

1. Chérie, op. cit., chap. LXXIX, p. 245. Le personnage est ensuite décrit : "Légèrement migrainée ce jour-là, elle étalait les grâces affaissées d'une Parisienne souffreteuse ". Migraine et chaise longue sont aussi associées après la fatigue que cause à Chérie la confection de sa première robe de soirée (chap. LIV, p. 184).

2. Ibid., chap. LXXVIII, p. 242.

3. Dans Chérie, Edmond se veut Peintre de la vie moderne. Le dialogue avec l'essai de Baudelaire est d'une extraordinaire richesse. Baudelaire lui-même a lu les Goncourt historiens d'art et leurs ćtudes sur les peintres de moeurs du XVIII' siècle, comme les Saint-Aubin qu'il cite dans sun premier chapitre, où il se comporte en collectionneur feuilletant un carton de gravures et espérant "la résurrection de ces costumes".

4. Chérie, op. cit., chap. LXXV, p. 239. Voir particulièrement la note 1, p. 239.

5. Ibid., chap. LXXVIII, p. 242. 
Mais Chérie retombe bientôt du flottement dans "le bleu d'élégances séraphiques " dans la matérialité la plus triviale : la meilleure preuve de cette négation du corps par la toilette, c'est son violent retour en opposant acharné, quand se déclare "un désir excentrique, une envie de choses toutes terrestres", le besoin de "manger des cornichons en buvant de la mauvaise bière". C'est en fait le dispositif duel de La Chambre double qu'Edmond de Goncourt reproduit ici, de la divinisation du corps féminin (opéré par la rêverie sur le costume et non plus sur le décor) au réveil d'une réalité matérielle (ici charnelle) plus sordide. Mais la fracture, thématisée par la violence du désir et le retour d'une temporalité impérative ("à l'improviste", "tout à coup"), est multiple: elle scinde d'abord la scène itérative de la contemplation (de la rêverie à l'irruption du désir charnel), elle renverse aussi sur le long terme l'évolution physiologique de la jeune fille (« il était venu à la jeune fille, tout à coup, comme cela, le goût dépravé de manger des cornichons de l'épicier d'à côté »), et marque le début d'une dégradation.

C'est pourquoi on va passer d'une toilette maîtrisée qui sublime le corps (même si elle ne parvient pas toujours à brider ses désirs), à une toilette dont les excès traduisent la désorganisation du corps, et qui révèle donc au lieu de nier. Ce dérèglement de la parure a été préparé avec l'apparition d'une nouvelle confidente de Chérie, Suzanne Malvezin dont le maquillage outrancier dénature le visage en même tant qu'il accuse violemment sa réalité charnelle :

La coquetterie de cette singulière jeune femme du monde, comment en bien exprimer la démence! Elle ne paraissait en quête que d'arrangements bizarres, de maquillages horrifiques, de détails de toilettes spectrales. Elle se faisait, à l'aide de la belladone, des regards ayant perdu leur caractère humain dans la largeur des pupilles [...]. Et elle travaillait [...] à la composition d'un visage aphrodisiaque et cadavéreux, où il y avait de l'échappée d'hôpital, mêlée à une espèce de génisse inquiète et fantasque $[. . .]^{\prime}$.

De tels excès sont interdits à Chérie, qui n'est pas, comme son amie, une femme mariée, et si elle atteint finalement à la même morbidesse (" des yeux de

1. Ibid., chap. LXV, p. 219-220. Voir aussi chap. LXXIX, p. 252 : "le teint tout farineux de poudre de riz, les lèvres comme saignantes de vermillon, les pupilles de la grandeur de pièces de quatre sous dans une noire cernure artificielle, les cheveux fulgurants, et le front entièrement mangé par les cheveux [...] ". Si les Goncourt vingt ans plus tôt étaient déjà sensibles à une esthétique de la morbidesse (voir Jean-Louis Cabanès, "Les Goncourt et la morbidité, catégorie esthétique de L'Art du XVIIf siècle ", Romantisme, n' 71,1991 ), Edmond témoigne ici d'un fait de mode (sur le maquillage des années 1875-1880, voir la note p. 220) et entend dégager un nouveau type social, lié à la pratique du spiritisme ("Suzanne Malvezin, la possédée, la détraquée, la toquée, présentait le plus parfait type de la malade morale du XIX siècle. ", p. 218), et désigné dès 1869 dans le Journal comme celui de la " nerveuse surexcitée " (note p. 219). 
fièvre dans le masque d'une figure cadavérique' "), celle-ci est moins composée que subie, sous l'effet de la maladie ; certes, croyant l'atténuer, elle l'accentuera à son tour, quand elle demande à sa femme de chambre de la maquiller pour aller une dernière fois au spectacle ("Allons, toi, mets-moi du rouge"): "dans sa pâleur, avec ses grands yeux extasiés et sa bouche sourieuse aux coins d'ombre, elle apparaissait comme une moribonde ${ }^{2}$ ". Bien avant d'arriver à cette agonie où la parure excessive révèle véritablement la mort qui ronge et la proche décrépitude du corps, les surenchères décoratives de Chérie l'ont quand même conduite à une sorte de dérèglement de la toilette, qui dit presque le retour du corps à l'état de nature :

Chérie se faisait coiffer par sa femme de chambre avec des gazons, de grandes herbes vertes tressées en couronne au-dessus de ses cheveux épars, et lui tombant jusqu'aux jarrets : coiffure qui scandalisait les rigides mères de famille, et d'où était venue à la petite fille du maréchal le surnom de l'échevelée ${ }^{3}$.

On voit bien ainsi comment la parure fonctionne selon un dispositif complexe d'occultation et de révélation d'un corps inquiétant, susceptible d'insidieuses comme de brutales métamorphoses, dont le personnage lui-même se fait le premier témoin, ou la première victime.

\section{Dédoublements, clivage, perte d'identité}

Dans le chapitre qui semble démarquer La chambre double, et proposer un corps double, sublimé par le costume ou dominé par le désir, la fracture intervient en fait bien plus tôt. Elle est présente au cœur même du dispositif euphorique qui fait l'éloge de la toilette, parce que Chérie parée est à la fois l'objet plastique contemplé et le créateur satisfait qui le contemple. Cette dualité, qui pourrait être positivement connotée si elle renvoyait à une sorte d'unité réalisée ${ }^{4}$, est ici fortement thématisée comme un clivage, et ce clivage ira croissant. Certes le fantasme d'une complétude est présent ("Chérie demeurait en sa chambre, en des adorations paresseuses de sa personne, dépensant de la coquetterie pour elle toute seule, et dérangée désagréablement par une visite $\left.[\ldots]^{5} »\right)$,

1. Ibid., chap. CI, p. 290.

2. Ibid., chap. CIII, p. 298, et CIV, p. 300.

3. Ibid., chap. LXXXI, p. 259.

4. Etre à soi-même une cuvre d'art, ou faire de sa vie une cuvre d'art : n'est-ce pas l'idéal qui sous-tend la posture du dandy ou de l'esthète de la fin de siècle ?

5. On est proche aussi de l'auto-érotisme qu'on observe plus loin avec le parfum (autre composante de la parure), et présent déjà chez Manette Salomon ou chez Nana. 
mais il est démenti d'emblée par la référence au théâtre, au jeu de l'actrice, au dédoublement de l'apparence dans un miroir :

Chérie avait adopté pour tenue de chambre cette toilette un peu théâtrale, mais qui lui allait divinement bien, et qui, reflétée dans sa psyché, lui montrait, au milieu d'éclairs brisés, un rien du rêve que toute femme fait à l'endroit de sa beauté'.

On ne négligera pas le motif du bris, qui annonce autant le rêve anéanti (réduit au "rien ") que l'identité durablement morcelée. De fait, le développement du motif de l'actrice confirme ce clivage, signe d'une dépossession :

Dans cette ambition cabotine des hommages universels, il lui arrivait de soigner, à la façon d'une actrice, ses entrées, et d'appartenir cœur et âme, tout le temps qu'elle passait dans le monde, à la production d'effets ${ }^{2}$.

L'existence entière de Chérie est placée sous le signe du théâtre, du rôle de grande personne que la fillette joue dans le chapitre d'ouverture à la scène finale où, mortellement malade, elle s'habille pourtant pour aller au spectacle. Mais c'est elle que la salle regarde; elle-même semble l'image au miroir de l'actrice sur scène, dans le rôle de Lucia di Lammermoor près de mourir; et quand à son tour elle regarde la salle, c'est pour y voir une spectatrice dont la situation reflète la sienne, une malade qui joue le jeu de la vie, et dont le corps doit être le lendemain coupé par le bistouri du chirurgien.

La scission de la personnalité de l'actrice, qui devient césure physique du corps, renvoie à un clivage psychologique essentiel, qu'on retrouve en fait dans d'autres expériences que celles de la toilette et de l'auto-contemplation effective, lesquelles transposent ce dispositif qui consiste à s'observer soi-même et à faire le constat de la distance, voire de la perte à soi-même. Chérie enfant a d'abord la perception d'un dédoublement de sa personnalité dans l'expérience du souvenir :

Il y avait également, dans ses souvenirs les plus lointains, une chute, une dégringolade du haut en bas d'un escalier, au pied duquel elle se voyait étendue sur le dos et s'entendait crier : "Je suis cassee, je suis cassée !" tout en ne se sentant aucun mal et n'osant cependant pas se relever toute seule. [...] Et Chérie se souvenait fort bien que tout en criant d'une voix larmoyante : " je suis cassée !" elle s'amusait beaucoup du voletage affolé [...] d'une pie que sa culbute avait effrayée.

1. Ibid., chap. LXXVIII, p. 242.

2. Ibid., chap. LXXIV, p. 237.

3. Ibid., chap. XVI, p. 107. 
Le souvenir de la prime enfance ne fait pas seulement apparaître deux êtres distincts, l'enfant grandie qui voit dans sa mémoire la petite fille du passé, il fait revivre une expérience de la scission au sens presque physique (la peur " d'être cassée "), et déjà réellement éprouvée sur le plan psychologique dans ce moment ancien, puisque ce qu'elle disait alors ne correspondait pas à ce qu'elle ressentait (" tout en ne se sentant aucun mal"), et qu'elle en avait conscience (" tout en criant [...], elle s'amusait beaucoup $")$.

Cette étrangeté à soi-même, Chérie en fait plus tard l'expérience dans la lecture et la musique, de manière moins inquiétante, parce que la sensation de la perte semble aussi accès à une vie plus intense, hors des limites de l'être :

Cette fusion de son être avec l'héroöne du livre la gratifiait d'une jouissance infinie, d'une absence d'elle-même ineffable, dans laquelle elle ne savait trop ni ce qui se passait autour d'elle, ni ce qu'on lui disait, ni même ce qu'elle répondait'.

Ici le personnage n'a pas simultanément la conscience critique de son dédoublement, et si Edmond de Goncourt présente la musique, suite naturelle du ravissement de la lecture, comme le "haschisch des femmes", il n'y associe pas la distance analytique par laquelle on prend acte de la dépossession vécue, le narrateur masculin conservant ainsi envers son personnage une position de maîtrise, d'autant plus nécessaire que la différenciation sexuelle s'amorce.

La métaphore du haschisch annonce d'autres hallucinations, celles de Chérie superstitieuse, et obsédée par sa difficulté à se marier. Elle essaie de voir en rêve son futur époux, mais la fatigue de l'insomnie et de la faim lui font prendre son linge à la lumière de la lune pour un linceul, préfiguration du cauchemar où elle se voit morte ${ }^{2}$. On voit donc que le roman combine ce qu'on a pu appeler « les phénomènes du sommeil " ", rêve, souvenir, hallucination (maladive ou suscitée par la lecture et la musique) et orchestrés ici non pas tant par les mises en scène du décor, et les projections qu'il autorise, que par celles du costume et les dédoublements qui en résultent, de sorte que l'identité de Chérie se diffracte en une liste de robes, en une liste de lieux mondains ${ }^{4}$, stylistique énumérative du

1. Ibid., chap. XXVII, p. 127.

2. Ibid., chap. XCII, p. 273-276.

3. Analysés par les médecins dès le milieu du XIX siècle, ils sont récurrents dans le roman psychologique de la fin de siècle. Voir Tony James, Vies secondes, Gallimard (Connaissance de l'inconscient), 1997 ; et ma communication "La chambre mentale" au colloque Médecine, sciences de la vie et littérature, sous la direction de Lise Dumasy et Hélène Spengler, Université Stendhal-Grenoble III, 13-15 mars 2008, à paraître.

4. Le chapitre LXVI (ibid., p. 221-223), inauguré par cette phrase nominale qui annonce bien la dispersion de la jeune fille ("En ces années 1867 et 1868, tous les jours d'hiver, des bals, des bals dont Chérie manqua à peine un seul. »), est exclusivement une liste de bals. Voir aussi chap. LXXIII, p. 2.35. 
morcellement que corrobore l'émiettement en courts chapitres centrés sur une situation et un vêtement spécifique (Chérie en écolière, en amazone, en patineuse, etc.), sans oublier que chaque robe est elle-même un assemblage de morceaux disjoints'.

Cependant le dispositif de sériation attaché à l'évocation des costumes n'a pas seulement pour but de suggérer une identité morcelée. Il entre aussi dans une dynamique inverse pour parer à un autre danger. Si l'identité psychologique de Chérie se présente comme clivée (en une expérience que le narrateur partage $e^{2}$, au contraire son corps (bien désigné cette fois comme le corps de l'autre $\left.{ }^{3}\right)$ semble une forme labile, insaisissable, en perpétuelle métamorphose :

Du bébé, du joli être grassouillet et rondelet, tout à l'heure enfoui dans l'envolement empesé d'une mousseline blanche au gros chou de soie bleu, commence à se dégager, en sa robe courte, en son droit tablier d'étude, en ses bas montrés jusqu'aux genoux, la petite fille longuette de sept ans ${ }^{4}$.

Le costume semble la chrysalide d'une mue dont il est difficile de cerner les étapes, sinon par la collection des toilettes successives. Dans l'évolution physiologique, c'est l'identité sexuelle elle-même qui est rendue incertaine, et c'est le vêtement qui semble la définir, comme lorsqu'il matérialise, dans la puberté, l'apparition des premières règles, avec " le passage de la petite robe courte à la grande robe $"$.

L'écriture de la liste aura donc aussi pour fonction de proposer, non seulement une collection de costumes selon les âges, mais aussi une typologie des différentes natures féminines à un âge donné. C'est pourquoi Edmond de Goncourt entoure son personnage d'autres jeunes filles qui sont autant de "types originaux de Parisiennes ». Dans le chapitre LXIV, la liste de jeunes filles repose

1. C'est ce que souligne exemplairement le chapitre consacré à la confection (ibid., chap. LIV, p. 184-187).

2. Voir le propos général qu'il tient d'abord sur le souvenir ou l'analogie qu'il s'attache à construire entre musique (pour les femmes) et usage de la drogue (pour les hommes), où la marque du discours d'époque nous importe moins que le parallélisme qui s'efforce tout à la fois de souligner et de gommer la différenciation sexuelle.

3. Aucun parallèle n'est esquissé avec l'évolution du jeune garçon. Les personnages masculins sont figés dans un âge et une situation donnés, hormis, à l'entrée du roman, le père de Chérie, rapidement évoqué de l'enfance à l'âge d'homme, mais cet être qui se métamorphose est aussi presque androgyne ; frontière des âges et frontière des sexes se fondent.

4. Ibid., chap. XIII, p. 101.

5. "Une métamorphose étrange : en ce corps de Chérie qui était, venons-nous de dire, la grâce même, depuis des mois se glissait quelque chose de laidement garçonnier. [...] Un être qui n'était plus une petite fille et pas encore une femme, un être au sexe comme indécis et non définitivement arrêté et en train de se chercher [...] " (ibid., chap. XXXVII, p. 149).

6. Ibid., chap. XL, p. 153. 
en outre sur des couples antithétiques, en une classification voyante : la grande et la petite (Germaine Dangirard, "grande fille de cinq pieds deux pouces", Andrée Cheylus, "jolie, mais dans l'infiniment petit ") ; la maniérée, à la féminité ostentatoire (Claire Dapogny, "le type et la personnification de la demoiselle-actrice") et la négligente (Emmeline Lauverjat, " une créature charpentée comme un homme ", qui a "le goût des exercices masculins"); la femme précoce, et la femme attardée dans l'enfance (Nanette Malhotier "sensuelle des pieds à la tête ", Blanche Champromain, " mignonne jeune personne donnant à voir la mine boudeuse d'un enfant qui fait ses dents"); la passionnée (Louise Neviance, " une bravoure dans les sentiments tout à fait rare ") et la superficielle (Lucie Préaudeau, "une vraie cervelle d'oisillon"). Il termine par deux êtres à part de la classification, mais en exceptions qui confirment sa validité, car il semble s'agir d'hybrides : l'Américaine, "mélange " d'entrain et de paresse, de connu et d'inconnu (Diana Peterson, "un amusant diable au corps de tout l'être, mélangé de la plus étonnante paresse à apprendre la langue française, [...] séduisante étrangère, habitant Paris depuis des années") ; et la "détraquée " (Suzanne Malvezin), finalement comparée aux "monstres féminins » de la mythologie scandinave.

Cependant ce quadrillage voyant semble mis à mal ; loin de fixer des types féminins, il suggère une instabilité de l'identité sexuelle. Non seulement des traits masculins sont présents dans la moitié des types évoqués, mais encore les plus féminins d'entre eux ne sont finalement qu'une façade, que contredit la fermeté du caractère :

Au fond, sous l'apparence de victime de la fillette, on rencontrait une volonté de fer, en même temps qu'une rouerie de vieux conseiller aulique sous son air intéressant de fausse poitrinaire'.

Outre le dispositif de la liste, il existe un autre moyen pour fixer les métamorphoses d'un corps labile : mis à part et observé de très près, à un moment précis de son évolution, il apparaît presque réifié. Quatre chapitres (LVII-LXI) vont tenter d'immobiliser Chérie à seize ans, en donnant successivement son portrait physique puis son portrait intellectuel, en limitant celui-ci au stéréotype, par la négative (Edmond de Goncourt refuse pour elle " une intelligence hommasse ", et insiste "sur les qualités féminines d'une intelligence qui ne sont pas les facultés de l'entendement masculin") puis de manière affirmative, en faisant de Chérie l'incarnation de la grâce. De la sorte, Chérie à seize ans est bibelot d'étagère (" elle était la femme article-paris, un être de goût, gentil

1. Ibid., chap. LXIV, p. 213. Emily Apter avait déjà remarqué ce problème de cross gender, cette incertitude de l'identité sexuelle dans les livres des Goncourt consacrés à des femmes du XVIII siècle ayant joué un rôle historique important (Feminizing the Fetish. Psychoanalysis and Narrative Obsession in Turn-of-the-Century France, Cornell University Press, 1991). 
comme la bimbeloterie qui porte ce nom ") ou pièce de collection ("Chérie était la femme qui n'est jamais tout le monde, Chérie était l'être rare "). Et on ne s'étonnera pas que ce "charmant et frêle objet d'art " trouve des prolongements ou des amorces dans le roman, sous la forme de la poupée, que la petite fille prépare, comme une projection d'elle-même, pour sa nuit de noce', ou du mannequin, qu'il s'agisse de ces femmes qui font le "métier de porte-manteau " chez le grand-couturier ${ }^{2}$, ou de celui qui accueille Georgette de Suzange debout alors que Chérie moribonde gît à terre .

Mais l'ambivalence quant à l'identité sexuelle n'est pas pour autant définitivement évacuée. Après les chapitres médians qui tentent de figer le personnage, Chérie à dix-huit ans, au faîte de sa maîtrise de la mode, semble investie d'une sorte de pouvoir, par lequel elle domine la haute société parisienne ("il était curieux d'observer chez les autres femmes la reconnaissance humble, écrasée, presque douloureuse, que leur imposait la supériorité de sa mise $"$ ), par lequel aussi elle s'élève à la dignité du créateur : Edmond de Goncourt lui reconnaît alors une " science " et un talent "d'artiste " (qui relèvent d'ordinaire du masculin, selon les archétypes dix-neuviémistes). Elle est donc tout à la fois le bel objet d'art féminin et l'artiste créateur qui le met en formes.

Identité psychologique, identité sexuelle et identité auctoriale se trouvent donc conjointement questionnés dans Chérie. Si le ton souvent péremptoire, les intrusions voyantes du romancier, affichent une assurance dans la conduite du

1. Chérie, op. cit., chap. LXXI, p. 233. Voir aussi Chérie sur le point de mourir : " la petite-fille du maréchal [...] tombe de côté, la tête sur le parquet, avec le bruit sec et la silhouette détraquée d'une poupée cassée en deux " (ibid., chap. CII, p. 293).

2. "de jeunes femmes lasses, aux traits fanés et fripés, des femmes ayant en quelque sorte perdu à leur métier de porte-manteau l'animation humaine, des femmes gagnées par l'automatisme du mannequin, promenaient sur leur dos mort des robes, des robes toutes vivantes et toutes lumineuses " (ibid., chap. LIII, p. 180)

3. Ibid., chap. CI, p. 290.

4. Ibid., chap. LXXV, p. 237.

5. "la toilette pour une femme [...] c'est le moyen de faire de sa personne [...] un charmant et frêle objet d'art, toujours renouvelé, toujours nouveau " (ibid., chap. LXXVI, p. 240). Cette force conquise par le personnage se manifeste aussi par l'indépendance qu'elle acquiert vis-à-vis de la figure paternelle, laquelle représente aussi le romancier (sur le plan symbolique comme sur le plan biographique, le vieil Edmond transposant ses relations avec la jeune Marie Abbatucci). Lorsque Chérie affirme (chap. LXIX, p. 229) : "Je ne veux pas me marier, monsieur mon grand-père ", on peut y voir un refus de la normalité sociale, visualisée par ces lieux en ordre que sont le cabinet de l'horticulteur et le salon du notaire où le vieil homme a essayé d'organiser une rencontre. Mais le cabinet de l'horticulteur est un emprunt direct au Journal, il renvoie à la passion de l'autcur, ailleurs mise en scène dans les hybridations florales, et il thématise à outrance le motif de la collection. Edmond semble jouir de la puissance conquise par son personnage à son détriment même. Sa rébellion, comme celle d'une Renée Mauperin, l'enchante, et il lui donne le dernier mot de ce chapitre. 
récit et dans la capacité à percer le mystère de "toute l'inconnue féminilité du tréfonds de la femme ", ils ne doivent pas masquer la façon dont la biographie, la psychologie et l'œuvre même de l'écrivain' se trouvent convoqués dans cet ultime roman qui est autant assomption du décoratif que questionnement sur les angoisses qu'il transpose.

Dominique PETY

1. On sait la part du biographique dans les premiers chapitres, qui empruntent beaucoup au propre père des Goncourt. Mais sur le plan bibliographique, il ne faut pas négliger le puissant jeu d'échos entre le roman et sa préface, qui intègre celle du Journal. Or la préface du Journal donne des deux frères un portrait qui semble celui de Chérie ("Nous ne nous cachons pas d'avoir été des créatures passionnées, nerveuses, maladivement impressionnables. "Chérie, préface, op. cit., p. 49 ; " La petite fille était impressionnable d'une manière extraordinaire. Une gronderie, le refus de quoi que ce soit, enfin un chagrin de son âge, un tout petit chagrin, la rendaient malade [...]" Chérie, chap. VII, p. 84) ; et les diaristes doivent, comme le romancier, tenter de capter le mouvant (" le changement indiqué chez les personnes qui nous furent familières ou chères, ne vient-il pas du changement qui s'était fait en nous ? "ibid., p. 48-49). D'autres jeux d'échos existent entre ce roman et ceux des deux frères, particulièrement dans l'avant-dernier chapitre qui condense l'agonie d'autres héroïnes des Goncourt: Germinie qui cache sa mort en se préoccupant des amours d'autrui, Élisa qui en revient aux souvenirs de son enfance dans la nature vosgienne, Philomène à travers la spectatrice qui attend le bistouri du chirurgien, Renée à laquelle ses parents vont survivre... 